BUTP-95/31

\title{
Quantum Field Theory on Certain Non-Globally Hyperbolic Spacetimes
}

\author{
C.J. Fewster"̈ and A. Higuchi市 \\ Institut für theoretische Physik, Universität Bern \\ Sidlerstrasse 5, CH-3012 Bern, Switzerland
}

June 14, 2018

\begin{abstract}
We study real linear scalar field theory on two simple non-globally hyperbolic spacetimes containing closed timelike curves within the framework proposed by Kay for algebraic quantum field theory on non-globally hyperbolic spacetimes. In this context, a spacetime $(M, g)$ is said to be 'F-quantum compatible' with a field theory if it admits a *-algebra of local observables for that theory which satisfies a locality condition known as 'F-locality'. Kay's proposal is that, in formulating algebraic quantum field theory on $(M, g)$, F-locality should be imposed as a necessary condition on the *-algebra of observables.

The spacetimes studied are the 2- and 4-dimensional spacelike cylinders (Minkowski space quotiented by a timelike translation). Kay has shown that the 4dimensional spacelike cylinder is F-quantum compatible with massless fields. We prove that it is also F-quantum compatible with massive fields and prove the Fquantum compatibility of the 2-dimensional spacelike cylinder with both massive and massless fields. In each case, F-quantum compatibility is proved by constructing a suitable F-local algebra.
\end{abstract}

PACS Numbers 04.62.+v

*E-mail address: fewster@butp.unibe.ch

${ }^{\dagger}$ E-mail address: higuchi@butp.unibe.ch 


\section{Introduction}

The theory of quantum fields in curved spacetime has been developed for the most part in the context of globally hyperbolic Lorentzian spacetimes. These spacetimes are distinguished by the fact that they contain Cauchy surfaces $\$$, which entails the global existence and uniqueness of certain fundamental solutions to the Klein-Gordon and Dirac equations (in particular the advanced-minus-retarded fundamental solution) which play a key rôle in the quantisation of these theories.

There has, however, been recent interest in quantum field theory on spacetimes containing closed timelike curves (CTCs), which do not possess Cauchy surfaces and for which the usual methodology must be modified. There are various reasons for this interest. For example, because such spacetimes are a 'source of tension' between quantum theory and general relativity, one would expect that they might be associated with or forbidden by non-trivial quantum gravitational effects (cf. Hawking's Chronology Protection Conjecture [2]). Even disregarding back-reaction, the formulation of quantum field theory in fixed background spacetimes containing CTCs raises many interesting conceptual problems stemming from the lack of a global Cauchy surface. As an example, we mention the fact that the evolution of interacting quantum fields from the far past of an isolated region of CTCs to its far future turns out to be non-unitary [3, 4, 5], which has led to a debate on how this can be interpreted [6] or alternatively, how unitarity may be restored [7, 8].

In the presence of such conceptual problems, there are clearly advantages in adopting a mathematically rigorous approach to quantum field theory in curved spacetime, such as that offered by the algebraic approach, even though this restricts us (at least for the moment) to the study of linear (non-self-interacting) field theories. The algebraic approach has also been developed largely in the globally hyperbolic case (see [1], 9] for detailed reviews). Recently, however, Kay [四] and Yurtsever [10 have independently proposed suitable generalisations of this approach to the non-globally hyperbolic case. Here, we will work within the framework developed by Kay. In this proposal, the aim is to construct a $*$-algebra $\mathcal{A}(M, g)$ whose elements are interpreted as polynomials in quantum fields smeared by smooth test functions compactly supported on a given spacetime $(M, g)$, and such that $\mathcal{A}(M, g)$ possesses the property of $F$-locality. This condition, which will be reviewed in Section 2, essentially requires that every point in $M$ should have a globally hyperbolic neighbourhood $N$ such that the induced algebra $\mathcal{A}(M, g ; N)$ (i.e., the subalgebra of $\mathcal{A}(M, g)$ consisting of polynomials of fields smeared by test functions supported in $N)$ should coincide with the intrinsic algebra $\mathcal{A}\left(N,\left.g\right|_{N}\right)$, obtained by regarding $\left(N,\left.g\right|_{N}\right)$ as a spacetime in its own right and following the normal procedure for globally hyperbolic spacetimes with some choice of time orientation on $N$. More precise definitions will be given in Section 2. If a spacetime admits an F-local *-algebra corresponding to a given field theory, the spacetime is said to be F-quantum compatible

\footnotetext{
${ }^{1}$ Following [1], we define a Cauchy surface in a Lorentzian spacetime to be a smooth spacelike surface intersected precisely once by each inextendible causal (time-like or null) curve contained in the spacetime. A spacetime is said to be globally hyperbolic if and only if it contains a Cauchy surface.
} 
with this field theory. Kay argues that non-F-quantum compatible spacetimes would not appear as classical approximations to states in quantum gravity. The proposal of Yurtsever [10] differs from that of Kay in that it constructs an algebra which generally fails to be F-local, except in the case of 'quantum benign' spacetimes [10].

Returning to Kay's proposal, it is clearly important to determine which spacetimes are F-quantum compatible with given field theories and which are not. Three results due to Kay [1] shed some light on this issue for both massive and massless real linear scalar field theory. Firstly, all globally hyperbolic spacetimes are F-quantum compatible with these theories; moreover, the usual minimal algebra on these spacetimes is F-local. Secondly, any subspacetime (of zero co-dimension) of a globally hyperbolic spacetime is also F-quantum compatible with these field theories. Thirdly, non-time orientable spacetimes are not F-quantum compatible with either field theory.

As well as these results, Kay discussed two examples of spacetimes with closed timelike curves. The first, 2-dimensional Misner space - in which a region of closed time-like curves 'develops' - was shown to be non-F-quantum compatible with massless scalar field theory. The second, the 4-dimensional 'spacelike cylinder' (i.e., Minkowski space quotiented by a fixed time translation) is F-quantum compatible with massless real linear scalar field theory. However, because (in both cases) the assumption of zero mass plays an essential rôle in his argument, Kay left the massive case as an open question.

In the present paper, we resolve this question for the four dimensional spacelike cylinder, which we show to be F-quantum compatible with massive real linear scalar field theory. We also demonstrate the F-quantum compatibility of the 2-dimensional spacelike cylinder with both massive and massless fields. If Our method in each of these cases is essentially to find a global bi-solution $\widetilde{\Delta}\left(x_{1} ; x_{2}\right)$ to the appropriate Klein-Gordon equation which plays the rôle of the advanced-minus-retarded fundamental solution, and which agrees with the Minkowski space fundamental solution for $x_{1}$ and $x_{2}$ sufficiently close together. Once this is found, it is easy to construct an F-local *-algebra for the corresponding real linear scalar field theory. Our construction is not unique: it turns out that there are many F-local algebras on the spacetimes we consider. As yet, it is not clear whether or not these different algebras correspond to different physics. To resolve this issue, one would have to study the physically 'nice' states (say, quasi-free and Hadamard) on these algebras, as suggested by Kay [1].

\section{Algebraic Quantum Field Theory and F-locality}

We start by briefly reviewing Kay's proposal [1] for the algebraic approach for real quantum field theory for the Klein-Gordon equation

$$
\left(\square_{g}+m^{2}\right) \phi=0
$$

\footnotetext{
${ }^{2}$ The massless case was also known to Kay (private communication).
} 
on non-globally hyperbolic, time orientable spacetimes $(M, g)$. This proposal is developed for the version of algebraic field theory in which the basic object is a $*$-algebra whose elements are interpreted as polynomials in smeared quantum fields. (Yurtsever adopts an alternative viewpoint, in which one considers the Weyl algebra associated with a suitable symplectic space of classical solutions - see, e.g., 99 - in his approach to quantisation on non-globally hyperbolic spacetimes [10].) In the following, $C_{0}^{\infty}(M)$ denotes the space of smooth, real-valued functions compactly supported on $M$.

We begin by defining a pre-field algebra to be a *-algebra with identity, $\mathbb{1}$, of polynomials over $\mathbb{C}$ in abstract objects $\phi(f)$ labelled by $f \in C_{0}^{\infty}(M)$ such that the following hold:

(Q1) Hermiticity: $(\phi(f))^{*}=\phi(f)$ for all $f \in C_{0}^{\infty}(M)$

(Q2) Linearity: $\phi\left(\lambda_{1} f_{1}+\lambda_{2} f_{2}\right)=\lambda_{1} \phi\left(f_{1}\right)+\lambda_{2} \phi\left(f_{2}\right)$ for all $\lambda_{i} \in \mathbb{R}, f_{i} \in C_{0}^{\infty}(M)$

(Q3) Field Equation: $\phi\left(\left(\square_{g}+m^{2}\right) f\right)=0$ for all $f \in C_{0}^{\infty}(M)$.

The motivation for this definition is the interpretation of $\phi(f)$ as the smearing by $f$ of a Hermitian weak solution $\phi(x)$ to (2.1), i.e.,

$$
\text { ' } \phi(f)=\int_{M} \phi(x) f(x) d \eta '
$$

where $d \eta=\left|\operatorname{det} g_{a b}\right|^{1 / 2} d^{n} x$ is the volume element. We emphasise the heuristic nature of this interpretation: there is no quantum field $\phi(x)$ as such underlying this approach.

There is a natural mapping between any two pre-field algebras on $(M, g)$ which identifies elements corresponding to the same polynomial in the smeared fields. If this mapping is an isomorphism, we say that the pre-field algebras are naturally isomorphic.

In general, a pre-field algebra does not represent a quantised field theory. If $(M, g)$ is globally hyperbolic, there exists a unique advanced-minus-retarded fundamental solution $\Delta\left(x_{1} ; x_{2}\right)$ to (2.1) (see, e.g., 12 ) and quantisation is effected by supplementing relations (Q1-3) with

(Q4) Covariant Commutation Relations: $\left[\phi\left(f_{1}\right), \phi\left(f_{2}\right)\right]=i \Delta\left(f_{1}, f_{2}\right) \mathbb{1}$ for all $f_{i} \in C_{0}^{\infty}(M)$,

where $\Delta\left(f_{1}, f_{2}\right)$ is the smeared version of $\Delta\left(x_{1} ; x_{2}\right)$ defined by

$$
\Delta\left(f_{1}, f_{2}\right)=\int_{M \times M} \Delta\left(x_{1} ; x_{2}\right) f\left(x_{1}\right) f\left(x_{2}\right) d \eta\left(x_{1}\right) d \eta\left(x_{2}\right) .
$$

We will refer to a pre-field algebra satisfying (Q4) as a field algebra. Such an algebra may be constructed as described in [1], by first forming the free *-algebra generated (over $\mathbb{C}$ and with $\mathbb{1}$ ) by the abstract objects $\left\{\phi(f) \mid f \in C_{0}^{\infty}(M)\right\}$ and then quotienting by the relations (Q1-4). We will refer to the algebra $\mathcal{A}(M, g)$ generated in this manner as the usual field algebra on $(M, g)$, when $(M, g)$ is globally hyperbolic. To be precise, 


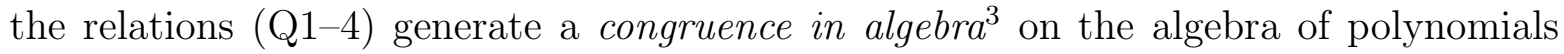
in the $\phi(f)$ and their adjoints, such that two polynomials are congruent if one can be manipulated into the form of the other using (Q1-4) and the usual rules of algebra. The quotient algebra $\mathcal{A}(M, g)$ is the algebra of congruence classes of polynomials. We will abuse notation by denoting a polynomial and its congruence class in the same way. For the most part, we will also suppress the mention of congruence classes.

For general non-globally hyperbolic manifolds $(M, g)$, there is no advanced-minusretarded fundamental solution and so one cannot define a field algebra in the above manner, although there may exist non-trivial pre-field algebras. Clearly, a criterion is required to select those pre-field algebras which correspond to some reasonable notion of quantum field theory. Kay proposed in [1] that this should be done as follows. Suppose $\mathcal{A}(M, g)$ is a pre-field algebra on a time orientable manifold $(M, g)$ and let $N$ be a globally hyperbolic subspacetime of $M$. There are two $*$-algebras naturally associated with $N$. Firstly, there is the induced *-algebra $\mathcal{A}(M, g ; N)$, namely, the subalgebra of $\mathcal{A}(M, g)$ consisting of polynomials in the $\phi\left(f_{i}\right)$ with all $f_{i}$ supported in $N$, along with the identity. $\mathcal{A}(M, g ; N)$ is easily seen to be a pre-field algebra on $\left(N,\left.g\right|_{N}\right)$. Secondly, because $\left(N,\left.g\right|_{N}\right)$ may be regarded as a globally hyperbolic spacetime in its own right, there is the intrinsic *-algebra, $\mathcal{A}\left(N,\left.g\right|_{N}\right)$ which is the usual field algebra on $\left(N,\left.g\right|_{N}\right)$. We require that quantisation in each region $N$ is carried out with respect to a time orientation induced from a fixed global time orientation on $(M, g)$. f The induced and intrinsic algebras are generally different (see [1] for explicit examples) because the commutator in $\mathcal{A}\left(N,\left.g\right|_{N}\right)$ is determined by the advanced-minus-retarded fundamental solution intrinsic to $\left(N,\left.g\right|_{N}\right)$ (and therefore depends on the causal structure of $\left(N,\left.g\right|_{N}\right)$ ), whilst that in $\mathcal{A}(M, g ; N)$ is induced from $\mathcal{A}(M, g)$ and may not respect the causal structure of $\left(N,\left.g\right|_{N}\right)$. However, Kay's proposal is that, as a necessary condition for $\mathcal{A}(M, g)$ to be a reasonable *-algebra for quantum field theory on $(M, g)$, these algebras should agree if $N$ is 'sufficiently small'. More precisely, this is codified by the notion of F-locality [1]:

Definition 2.1 (The F-locality Condition) Let $(M, g)$ be a (not necessarily globally hyperbolic) time orientable spacetime and suppose $\mathcal{A}(M, g)$ is a pre-field algebra for real linear scalar field theory on $(M, g) . \mathcal{A}(M, g)$ is said to be F-local if each point $p$ of $M$ has a globally hyperbolic neighbourhood $N$ for which the induced $\mathcal{A}(M, g ; N)$ and intrinsic $\mathcal{A}\left(N,\left.g\right|_{N}\right)$ *-algebras are naturally isomorphic. In addition, $(M, g)$ is said to be F-quantum compatible if it admits a pre-field algebra satisfying F-locality.

In [1], Kay established various consequences of this definition, including the result that the usual field algebra on a globally hyperbolic spacetime is F-local. Thus F-locality provides a generalisation of the usual quantisation procedure. It is important to note

\footnotetext{
${ }^{3} \mathrm{~A}$ congruence in algebra is a linear equivalence relation which respects the algebraic operations. Thus in our case, if the polynomials $P$ and $Q$ are congruent to $P^{\prime}$ and $Q^{\prime}$ respectively, then the product $P Q$ is congruent to $P^{\prime} Q^{\prime}$.

${ }^{4}$ In [1], Kay initially left open the possibility of the time orientation differing in different regions $N$. However, he then showed that time orientability was a necessary condition for F-quantum compatibility of a manifold: we have essentially incorporated this result into our discussion.
} 
that, even for F-local algebras, it is not the case that the induced and intrinsic algebras coincide for any subspacetime $N$ - see [1] for discussion.

We now describe the technique we will use to prove the F-quantum compatibility of the spacelike cylinder spacetimes in Section 3. This is accomplished by constructing a global bi-solution $\widetilde{\Delta}\left(x_{1} ; x_{2}\right)$ to the Klein-Gordon equation which is equal to the intrinsic advanced-minus-retarded fundamental solution on sufficiently small globally hyperbolic subspacetimes (i.e., for $x_{1}$ and $x_{2}$ sufficiently close together). We then use $\widetilde{\Delta}$ to define an F-local *-algebra.

More formally, we define:

Definition 2.2 Let $(M, g)$ be a (not necessarily globally hyperbolic) time orientable spacetime, and suppose that $\widetilde{\Delta}\left(x_{1} ; x_{2}\right)$ is an antisymmetric, real-valued global bi-solution to the homogenous Klein-Gordon equation on $M \times M$. We call $\widetilde{\Delta}$ an F-local advancedminus-retarded fundamental solution on $(M, g)$ if every point $p \in M$ has a globally hyperbolic neighbourhood $N$ such that the restriction of $\widetilde{\Delta}$ to $N \times N$ agrees with the intrinsic advanced-minus-retarded fundamental solution on $\left(N,\left.g\right|_{N}\right)$.

Here, the property of antisymmetry (i.e., $\left.\widetilde{\Delta}\left(x_{1} ; x_{2}\right)=-\widetilde{\Delta}\left(x_{2} ; x_{1}\right)\right)$ is required because we would like to regard $\widetilde{\Delta}$ (suitably smeared) as $-i$ times the commutator of smeared fields.

With this definition, the following is then almost immediate:

Theorem 2.3 If a spacetime $(M, g)$ admits an F-local advanced-minus-retarded fundamental solution $\widetilde{\Delta}$ to the Klein-Gordon equation, then it is F-quantum compatible with real linear scalar field theory. Moreover, the algebra $\mathcal{A}(M, g)$ defined as the free *-algebra generated by the $\phi(f)$ for $f \in C_{0}^{\infty}(M)$ quotiented by the relations (Q1)-(Q3) and

$$
\left(Q 4^{\prime}\right) \text { Modified CCRs: }\left[\phi\left(f_{1}\right), \phi\left(f_{2}\right)\right]=i \widetilde{\Delta}\left(f_{1}, f_{2}\right) \mathbb{1} \text { for all } f_{i} \in C_{0}^{\infty}(M)
$$

is F-local, where $\widetilde{\Delta}\left(f_{1}, f_{2}\right)$ is the smeared version of $\widetilde{\Delta}\left(x_{1} ; x_{2}\right)$.

Proof: Let $p$ be an arbitrary point in $M$ and let $N$ be a globally hyperbolic neighbourhood of the type guaranteed by Definition 2.2. Then the F-locality of $\widetilde{\Delta}$ implies that the natural mapping between $\mathcal{A}\left(N,\left.g\right|_{N}\right)$ and $\mathcal{A}(M, g ; N)$ is an isomorphism, because the relations $(\mathrm{Q} 1-3)$ and $\left(\mathrm{Q} 4^{\prime}\right)$ for $(M, g)$ agree with the relations $(\mathrm{Q} 1-4)$ for $\left(N,\left.g\right|_{N}\right)$ on polynomials involving only test functions supported in $N$.

Thus it suffices to exhibit an F-local advanced-minus-retarded fundamental solution on a spacetime $(M, g)$ in order to conclude its F-quantum compatibility and to construct an F-local algebra there.

In addition to F-locality, we will require that our *-algebra $\mathcal{A}(M, g)$ should be globally covariant with respect to the isometries of the spacetime (cf. [11]). That is, we 
require there to be a representation $\kappa \mapsto \alpha_{\kappa}$ of the isometry group of $(M, g)$ as automorphisms on $\mathcal{A}(M, g)$ such that $\alpha_{\kappa}(\phi(f))=\phi(f \circ \kappa)$ for each $f \in C_{0}^{\infty}(M)$ and isometry $\kappa$. This places constraints on the F-local advanced-minus-retarded fundamental solution $\widetilde{\Delta}\left(x_{1} ; x_{2}\right)$, because we have

$$
i \widetilde{\Delta}(f \circ \kappa, g \circ \kappa) \mathbb{1}=\alpha_{\kappa}(i \widetilde{\Delta}(f, g) \mathbb{1})
$$

for all $f, g \in C_{0}^{\infty}(M)$. Thus we require $\widetilde{\Delta}\left(\kappa\left(x_{1}\right) ; \kappa\left(x_{2}\right)\right)= \pm \widetilde{\Delta}\left(x_{1} ; x_{2}\right)$ for each isometry $\kappa$, with the sign depending on whether $\alpha_{\kappa}$ is a linear $(+)$ or anti-linear $(-)$ automorphism.

\section{Quantum Field Theory on Spacelike Cylinders}

In this section, we discuss the F-quantum compatibility of the 2- and 4-dimensional spacelike cylinders, which are obtained as quotients of 2- or 4-dimensional flat Minkowski space by a fixed time-like translation. We write coordinates for these spacetimes in the form $(t, \mathbf{x})$ and, in the 2-dimensional case, write the single spatial coordinate as $z$. The spacelike cylinder spacetime is then defined to be the quotient of Minkowski space by the fixed time translation $t \rightarrow t+T$ for some $T>0$.

In the following, it will be useful to have an explicit class of globally hyperbolic neighbourhoods available: accordingly, we define a diamond of size $d$ about position $(t, \mathbf{x})$ to be the region

$$
N_{d}=\left\{\left(t^{\prime}, \mathbf{x}^{\prime}\right)|| t^{\prime}-t|+| \mathbf{x}^{\prime}-\mathbf{x} \mid<d\right\} .
$$

In our example spacetimes every point has a globally hyperbolic diamond neighbourhood of any size less than $T / 2$. If a diamond neighbourhood in one of the spacelike cylinders is globally hyperbolic, its intrinsic advanced-minus-retarded fundamental solution is just the restriction of that for 2- or 4-dimensional Minkowski space as appropriate.

\subsection{The 2-dimensional Spacelike Cylinder}

We now show that the 2-dimensional spacelike cylinder spacetime is F-quantum compatible with both massless and massive real linear scalar field theory, by exhibiting suitable F-local advanced-minus-retarded fundamental solutions.

As mentioned above, we will require global covariance with respect to the isometries of the spacetime: these are generated by the group of spacetime translations and the two discrete symmetries of parity and time reversal. The translations form a continuous group of isometries and must therefore be implemented by linear automorphisms at the algebraic level, whilst parity and time reversal are implemented as usual by linear and anti-linear automorphisms respectively (locally, this is actually a consequence of F-locality). In consequence, $\widetilde{\Delta}\left(x_{1} ; x_{2}\right)$ can be written as a function of $x_{1}-x_{2}$ alone (by translational invariance): $\widetilde{\Delta}\left(x_{1} ; x_{2}\right)=\widetilde{\Delta}\left(t_{1}-t_{2}, z_{1}-z_{2}\right)$, where $\widetilde{\Delta}(t, z)$ is even in $z$ and odd in $t$. 
We now prove the F-quantum compatibility of the 2-dimensional spacelike cylinder, treating the massless and massive cases separately.

\section{Massless Klein-Gordon Theory}

It is helpful to begin by recalling the definition of the advanced-minus-retarded fundamental solution to the massless Klein-Gordon equation on 2-dimensional Minkowski space. This is given by $\Delta\left(t_{1}, z_{1} ; t_{2}, z_{2}\right)=\Delta\left(t_{1}-t_{2}, z_{1}-z_{2}\right)$, where $\Delta(t, z)$ solves

$$
\left(\frac{\partial^{2}}{\partial t^{2}}-\frac{\partial^{2}}{\partial z^{2}}\right) \Delta(t, z)=0
$$

with initial data

$$
\Delta(0, z)=0,\left.\quad \frac{\partial \Delta}{\partial t}(t, z)\right|_{t=0}=-\delta(z) .
$$

As is well known, the solution is

$$
\Delta(t, z)=-\frac{1}{2} \varepsilon(t) \vartheta\left(t^{2}-z^{2}\right)
$$

where $\vartheta(x)$ is the Heaviside function, and $\varepsilon(x)$ is the sign of $x$. Thus $\Delta(t, z)$ is equal to $-\frac{1}{2}$ in the future light-cone of the origin, $\frac{1}{2}$ in the past light-cone and vanishes elsewhere.

To construct an F-local advanced-minus-retarded fundamental solution on the spacelike cylinder, we regard it as the strip $|t|<T / 2$ in Minkowski space and impose periodic boundary conditions on all fields at $\pm T / 2$. We seek a solution $\widetilde{\Delta}$ to (3.2) which is periodic in time with period $T$ and which agrees with $\Delta(t, z)$ for $t, z$ sufficiently close to the origin. There are many possibilities for $\widetilde{\Delta}$ (see the corresponding discussion for the massive case); we give the simplest, which is

$$
\widetilde{\Delta}(t, z)=\sum_{n=-\infty}^{\infty}(-1)^{n} \Delta(t, z-n T / 2) .
$$

The sum converges (because only finitely many summands are non-zero at any given $(t, z))$ and its values are displayed in Figure 1. Note that $\widetilde{\Delta}$ is periodic in both $t$ and $z$ with period $T$, so it suffices to show the values for $|t|,|z|<T / 2$. $\widetilde{\Delta}$ is clearly the solution to (3.2) with initial data

$$
\widetilde{\Delta}(0, z)=0,\left.\quad \frac{\partial \widetilde{\Delta}}{\partial t}(t, z)\right|_{t=0}=\sum_{n=-\infty}^{\infty}(-1)^{n+1} \delta(z-n T / 2) .
$$

One may also regard $\widetilde{\Delta}(t, z)$ as the result of 'wrapping' the Minkowski space fundamental solution $\Delta$ round a spacelike cylinder of period $T / 2$ with anti-periodic boundary conditions. It is interesting to compare this with Kay's proof of F-quantum compatibility for massless fields on the 4-dimensional spacelike cylinder, which proceeds by wrapping the Minkowski fundamental solution round the cylinder of period $T$ with periodic boundary conditions. 


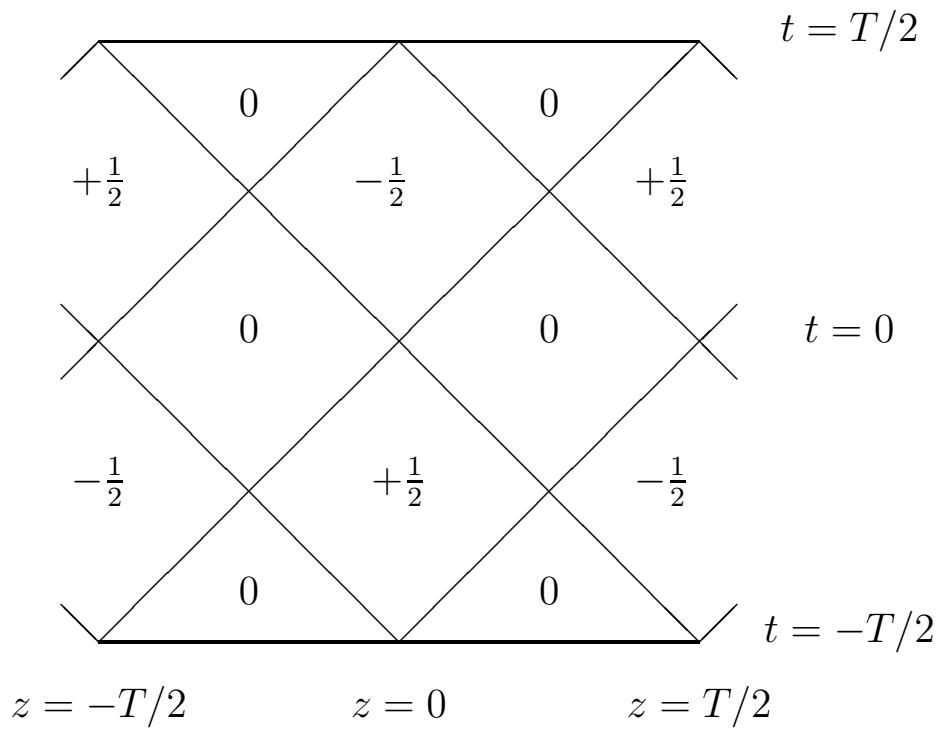

Figure 1: The values taken by the function $\widetilde{\Delta}(t, z)$ on the spacelike cylinder.

Finally, it is easy to see from Fig. 1 that $\widetilde{\Delta}\left(t_{1}, z_{1} ; t_{2}, z_{2}\right)=\widetilde{\Delta}\left(t_{1}-t_{2}, z_{1}-z_{2}\right)$ agrees with $\Delta\left(t_{1}, z_{1} ; t_{2}, z_{2}\right)$ within any diamond neighbourhood of size less than $T / 4$ about any point $p$ in the spacetime. (Note that if two points lie in a diamond region of size $d$ of some point, then their difference lies in a diamond region of size $2 d$ about the origin.) Thus $\widetilde{\Delta}$ is an F-local advanced-minus-retarded fundamental solution on the two dimensional spacelike cylinder. As an immediate corollary, due to the periodicity of $\widetilde{\Delta}$ in both $t$ and $z$, we also conclude F-quantum compatibility of the 2-torus with equal periods with massless field theory.

\section{Massive Klein-Gordon Theory}

In this case, we seek a global solution $\widetilde{\Delta}(t, z)$ to the massive Klein-Gordon equation on the spacelike cylinder, which agrees with the usual advanced-minus-retarded fundamental solution on 2-dimensional Minkowski space

$$
\Delta(t, z)=-\frac{1}{2} \varepsilon(t) \vartheta\left(t^{2}-z^{2}\right) J_{0}\left(m\left(t^{2}-z^{2}\right)^{1 / 2}\right)
$$

for $t$ and $z$ sufficiently close to the origin. Here, $J_{0}(z)$ is the Bessel function of order zero. As mentioned above, we will require $\widetilde{\Delta}(t, z)$ to be even in $z$ and odd in $t$.

The key to constructing such a function is that the initial value problem is well-posed for the Klein-Gordon equation on the spacelike cylinder, when one specifies initial data

$$
f(t)=\phi(t, 0) \quad \text { and } \quad g(t)=\left.\frac{\partial \phi}{\partial z}(t, z)\right|_{z=0}
$$

on the circle $z=0$, where $f(t)$ and $g(t)$ are periodic with period $T$. (Essentially, we are just turning the spacelike cylinder on its end to obtain the globally hyperbolic 'timelike 
cylinder', with $t$ regarded as a periodically identified 'spatial' coordinate and $z$ regarded as 'time'.)

We now set $g(t)$ to be identically zero (reflecting the fact that we want $\widetilde{\Delta}(t, z)$ to be even in $z$ ), and require that $f(t)$ should coincide with $\Delta(t, 0)=-\frac{1}{2} \varepsilon(t) J_{0}(m|t|)$ for $|t|<T / 2-\epsilon$ for some $\epsilon>0$. Outside this region, we can choose $f(t)$ arbitrarily, subject to the requirements that it be real-valued, odd, smooth and obey periodic boundary conditions at $t= \pm T / 2$. From elementary properties of hyperbolic equations, it follows that the solution $\widetilde{\Delta}(t, z)$ to the massive Klein-Gordon equation with this initial data agrees with $\Delta(t, z)$ in the diamond of size $T / 2-\epsilon$ about the origin. Thus $\widetilde{\Delta}\left(x_{1} ; x_{2}\right)=$ $\widetilde{\Delta}\left(t_{1}-t_{2}, z_{1}-z_{2}\right)$ is an F-local advanced-minus-retarded fundamental solution for the massive Klein-Gordon equation on the spacelike cylinder.

There is clearly a substantial non-uniqueness in our construction, because $f(t)$ can be chosen freely (amongst smooth, odd functions) for $T / 2-\epsilon<|t|<T / 2$ (at $t=T / 2$, $f$ must vanish, along with all its even derivatives). Thus there are very many F-local algebras on this spacetime. However, in general, the corresponding $\widetilde{\Delta}(t, z)$ functions are not bounded as $|z| \rightarrow \infty$ for each $t$, owing to the presence of exponentially growing modes in the solution to the initial value problem (3.8). [

To see this explicitly, note that mode solutions to the massive Klein-Gordon equation

$$
\left(\frac{\partial^{2}}{\partial t^{2}}-\frac{\partial^{2}}{\partial z^{2}}+m^{2}\right) \phi(t, z)=0
$$

with periodic time dependence $e^{2 \pi i n t / T}$ are $\phi_{n, \pm}(t, z) \propto e^{2 \pi i n t / T \pm i k_{n} z}$, where $k_{n}$ is determined by

$$
k_{n}=\left(\frac{4 \pi^{2} n^{2}}{T^{2}}-m^{2}\right)^{1 / 2} .
$$

Accordingly, for any $m>0$ there is at least one mode (solution with imaginary $k_{n}$ ) which grows exponentially as $|z| \rightarrow \infty$.

Presumably, one should exclude solutions with unbounded $\widetilde{\Delta}(t, z)$. One can arrange this by using the freedom in $f(t)$ to ensure that it possesses no harmonics corresponding to growing modes. Defining

$$
\ell_{n}(f)=\int_{-T / 2}^{T / 2} e^{2 \pi i n t / T} f(t) d t
$$

for $n \in \mathbb{Z}$, this is equivalent to requiring $\ell_{n}(f)=0$ for $|n| \leq m T / 2 \pi$. We note that the $n=0$ harmonic vanishes in any case because $f(t)$ is odd; thus for $m T<2 \pi$ (small cylinders or light particles) growing modes are automatically absent.

More generally, because $f(t)$ is chosen to be real-valued and odd, we have $\ell_{-n}(f)=$ $-\ell_{n}(f)=\ell_{n}(f)^{*}$, and so growing modes are excluded provided that $\ell_{n}(f)=0$ for

\footnotetext{
${ }^{5}$ This is the penalty for switching the interpretations of $t$ and $z$ : the 'mass' becomes imaginary!
} 
$1 \leq n \leq m T / 2 \pi$. This can be arranged by choosing $f(t)$ to have the form

$$
f(t)=-\frac{1}{2} \varepsilon(t) J_{0}(m|t|) \chi(t)+\sum_{1 \leq k \leq m T / 2 \pi} \lambda_{k} h_{k}(t),
$$

with $\lambda_{k} \in \mathbb{R}$, where $\chi(t)$ is smooth, even, compactly supported in $|t|<T / 2-\epsilon / 2$, and equal to unity for $|t|<T / 2-\epsilon$, and the $h_{k}(t)$ are smooth odd functions compactly supported in $T / 2-\epsilon / 2<|t|<T / 2$ such that $\ell_{j}\left(h_{k}\right)=0$ for $0 \leq j<k$ and $\ell_{k}\left(h_{k}\right) \neq 0$. The functions $\chi$ and $h_{k}$ are also required to be real-valued.

To construct suitable $h_{k}$, we define the function spaces

$$
\mathcal{D}_{n}=\left\{\varphi \in \mathcal{D}_{0} \mid \ell_{j}(\varphi)=0 \text { for } j=1,2, \ldots, k\right\}
$$

where $\mathcal{D}_{0}$ is the space of smooth odd real-valued functions compactly supported in $T / 2-\epsilon / 2<|t|<T / 2$. For each $k, h_{k}$ is chosen to be a representative of any non-zero equivalence class in the quotient $\mathcal{D}_{k-1} / \mathcal{D}_{k}$, which is non-trivial because $\ell_{1}, \ldots, \ell_{k}$ are linearly independent distributions on $\mathcal{D}_{0}$. The $h_{k}$ clearly possess the required properties and the $\lambda_{k}$ may therefore be chosen to ensure that $\ell_{n}(f)=0$ for each $|n| \leq m T / 2 \pi$ as required. Reality of the $\lambda_{k}$ follows from the fact that $\ell_{n}(\varphi)$ is purely imaginary for odd real-valued $\varphi$.

One could, of course, specify a distinguished $\widetilde{\Delta}$ by letting $\epsilon$ shrink to zero, thereby putting all the ambiguity in $f(t)$ into a distributional contribution supported at the point $t= \pm T / 2$. The distinguished $\widetilde{\Delta}$ would be obtained by requiring this distribution to be as 'regular' as possible, but nonetheless singular enough to ensure the absence of growing modes. In fact, the F-local advanced-minus-retarded solution identified in the massless case (where there are in fact no growing modes) can be regarded as a (trivial) example of this procedure.

\subsection{The 4-dimensional Spacelike Cylinder}

As we have mentioned, it has already been shown by Kay [1] that the 4-dimensional spacelike cylinder is F-quantum compatible with massless scalar fields. Here, we demonstrate that the same is true for the massive case.

The isometries of the 4-dimensional spacelike cylinder are generated by the group of spacetime translations, the group of rotations, and the discrete symmetries of parity and time reversal. Of these generators, only time reversal is represented by an anti-linear automorphism. The requirement of covariance with respect to these isometries therefore entails that $\widetilde{\Delta}\left(x_{1} ; x_{2}\right)=\widetilde{\Delta}\left(t_{1}-t_{2},\left|\mathbf{x}_{1}-\mathbf{x}_{2}\right|\right)$, where $\widetilde{\Delta}(t, r)$ is odd in $t$.

In addition, we require that $\widetilde{\Delta}\left(x_{1} ; x_{2}\right)$ should agree with the advanced-minus-retarded fundamental solution for 4-dimensional Minkowski space given by $\Delta\left(x_{1} ; x_{2}\right)=\Delta\left(t_{1}-\right.$ $\left.t_{2},\left|\mathbf{x}_{1}-\mathbf{x}_{2}\right|\right)$, where

$$
\Delta(t, r)=m \varepsilon(t) \vartheta\left(t^{2}-r^{2}\right) \frac{J_{1}\left(m\left(t^{2}-r^{2}\right)^{1 / 2}\right)}{4 \pi\left(t^{2}-r^{2}\right)^{1 / 2}}-\frac{\varepsilon(t)}{2 \pi} \delta\left(t^{2}-r^{2}\right) .
$$


The essential point here is that spherical symmetry has reduced this to an effectively 2-dimensional problem. Writing $\widetilde{\Delta}(t, r)=u(t, r) / r, u(t, r)$ satisfies

$$
\left(\frac{\partial^{2}}{\partial t^{2}}-\frac{\partial^{2}}{\partial r^{2}}+m^{2}\right) u(t, r)=0
$$

and we may proceed much as in Section 3.1, by specifying initial data on the circle $r=0$, defined by

$$
f(t)=u(t, 0) \quad \text { and } \quad g(t)=\left.\frac{\partial u}{\partial r}(t, r)\right|_{r=0} .
$$

We impose data with $f(t)$ identically zero and $g(t)$ given by

$$
g(t)=m \varepsilon(t) \frac{J_{1}(m t)}{4 \pi t}+\frac{\delta^{\prime}(t)}{2 \pi} \quad \text { for } \quad|t|<T / 2-\epsilon
$$

for some $\epsilon>0$. For $t$ outside this region, $g(t)$ is chosen to be smooth, odd and to obey periodic boundary conditions at $t= \pm T / 2$. We also use some of this freedom to ensure the absence of tachyonic modes in the same manner as before.

These data agree with the corresponding quantities obtained from the flat spacetime fundamental solution $\Delta(t, r)$ for $|t|<T / 2-\epsilon$ as may be seen by a simple distributional calculation, which rests on the following easily established result: Let $\psi_{r}(t)=r \varepsilon(t) \delta\left(t^{2}-\right.$ $r^{2}$ ) be a family of distributions labelled by $r$. Then

(a) $\psi_{r}(t)=\frac{1}{2}(\delta(t-r)-\delta(t+r)) \rightarrow 0$ as $r \rightarrow 0^{+}$

(b) $\left(\partial \psi_{r} / \partial r\right)(t)=-\frac{1}{2}\left(\delta^{\prime}(t-r)+\delta^{\prime}(t+r)\right) \rightarrow-\delta^{\prime}(t)$ as $r \rightarrow 0^{+}$.

Thus, the solution $\widetilde{\Delta}(t, r)$ obtained from this initial data agrees with $\Delta(t, r)$ for $|t|+r<T / 2-\epsilon$. Thus $\widetilde{\Delta}\left(x_{1} ; x_{2}\right)=\widetilde{\Delta}\left(t_{1}-t_{2},\left|\mathbf{x}_{1}-\mathbf{x}_{2}\right|\right)$ agrees with $\Delta\left(x_{1} ; x_{2}\right)$ when $x_{1}, x_{2}$ lie in a diamond neighbourhood of size $T / 4-\epsilon / 2$ of any given point, so $\widetilde{\Delta}$ is an F-local advanced-minus-retarded fundamental solution to the massive Klein-Gordon equation on the 4-dimensional spacelike cylinder. This spacetime is therefore F-quantum compatible with massive real linear scalar field theory. As in the 2-dimensional case, our construction is highly non-unique: similar comments to those made in that case also apply here. We also note that by setting $m=0$ in the above, we obtain a new proof of the F-quantum compatibility of this spacetime with massless field theory.

\section{Conclusion}

We have studied the 2- and 4-dimensional spacelike cylinders and have shown that the 2-dimensional cylinder is F-quantum compatible with both massive and massless fields, and that the 4-dimensional cylinder is F-quantum compatible with massive fields. 
This complements the earlier result of Kay on the F-quantum compatibility of the 4dimensional spacelike cylinder with massless fields and resolves the question raised in [1] as to whether or not this spacetime is F-quantum compatible with massive fields. These results help to strengthen the claim that F-locality provides a good basis for quantum field theory on non-globally hyperbolic spacetimes.

Our construction exhibits a considerable degree of non-uniqueness. A similar phenomenon was noted by Kay for spacetimes which can be isometrically embedded in a globally hyperbolic spacetime: here we have shown that this can happen even for spacetimes which cannot be embedded in this way. Non-uniqueness might present a serious problem if it transpired that different F-local algebras could correspond to different physics. To investigate this, it would be necessary to move beyond the construction of the algebra of observables, to the study of physically reasonable states on the algebra. If necessary, it might be possible to remove this non-uniqueness by imposing further conditions on the algebra of observables, or on the class of allowed states, as mentioned in [1].

Finally, our treatment of the spacelike cylinders relied heavily on the special property that they may be turned on end to yield globally hyperbolic spacetimes. More generally, it is of interest to determine what conditions a spacetime must satisfy in order to admit an F-local advanced-minus-retarded fundamental solution. This appears to be closely related to whether or not a spacetime is 'classically benign' [13 with respect to weak solutions of the Klein-Gordon equation. We note that (almost by definition) a translationally invariant classically benign spacetime admits an F-local advanced-minusretarded fundamental solution, and is therefore F-quantum compatible. More generally, one needs to generalise the notion of classical benignity to treat bi-solutions. Progress in the classification of such manifolds would be of considerable interest in the context of F-locality.

Acknowledgments: We are grateful to Bernard Kay, Petr Hájíček and Peter Minkowski for useful conversations and comments. CJF thanks the Royal Society and we both thank the Schweizerischer Nationalfonds for financial support. 


\section{References}

[1] B.S. Kay, Rev. Math. Phys. Special Issue (1992) 167

[2] S.W. Hawking, Phys. Rev. D46 (1992) 603

[3] D.G. Boulware, Phys. Rev. D46 (1992) 4421

[4] J.L. Friedman, N.J. Papastamatiou and J.Z. Simon, Phys. Rev. D46 (1992) 4456

[5] H.D. Politzer, Phys. Rev. D46 (1992) 4470

[6] S.W. Hawking, Quantum Coherence and Closed Timelike Curves

DAMTP-R95/4, gr-qc/9502017

[7] A. Anderson, Phys. Rev. D51 (1995) 5707

[8] C.J. Fewster and C.G. Wells, Unitarity of Quantum Theory and Closed Time-like Curves, DAMTP-R94/35, hep-th/9409156

[9] B.S. Kay and R.M. Wald, Physics Reports 207 (1991) 49

[10] U. Yurtsever, Class. Quantum Grav. 11 (1994) 999

[11] J. Dimock, Commun. Math. Phys. 77 (1980) 219

[12] A. Lichnerowicz, Publ. IHES no. 10 (1961)

[13] U. Yurtsever, J. Math. Phys. 31 (1990) 3064 